\title{
Interpretações do Interferômetro de Mach-Zehnder no Modelo qMG
}

R.B. AMARAL ${ }^{1}$, R.H.S. REISER ${ }^{2}$, A.C.R. COSTA ${ }^{3}$, Programa de Pós-Graduação em Informática, CP/UCPel, 96010-000 Pelotas, RS, Brazil.

\begin{abstract}
Resumo. Este trabalho analisa as interpretações de algoritmos quânticos no modelo qGM (Quantum Geometric Machine Model), para compreensão da construção da informação durante o processo de evolução dos sistemas quânticos. A estrutura ordenada do modelo qGM mostra-se capaz de interpretar a construção dos processos e dos estados quânticos baseada na concepção de objetos parciais, considerando a relação de inclusão como a ordem de informação. O trabalho apresenta estudo de caso, onde a interpretação de objetos parciais possibilita uma descrição contextualizada para o Interferômetro de Mach-Zenhder, relacionado com o fenômeno da interferência. Verifica-se que esta interpretação não pode ser obtida fora do esquema conceitual da Teoria dos Domínios.
\end{abstract}

Palavras-chave. Quantum Geometric Machine, Interferômetro Quântico, Computação Quântica.

\section{Introdução}

A versão quântica do modelo de Máquina Geométrica - indicado por qGM - está fundamentada na Teoria dos Domínios, mais especificamente nos domínios qualitativos, introduzidos por Girard [4]. O modelo qGM provê interpretação para estados e processos, possivelmente infinitos, considerando uma estrutura espacial da memória, com valores (complexos normalizados) indexados por elementos (base computacional) do conjunto dos ordinais transfinitos. A construção do domínio de estados $\mathbb{S}_{\infty}$ e de processos $\mathbb{D}_{\infty}$ é obtida em níveis, modelando as possíveis dimensões de um sistema quântico. A computação é concebida como uma transição de estados associada a uma localização espacial, obtida a partir da sincronização de processos quânticos, caracterizando a unidade de tempo computacional. O paralelismo quântico é caracterizado pela sincronização de processos elementares quânticos. Com base na representação parcial associada aos objetos do modelo qGM, o trabalho mostra a evolução de estados e portas quânticas referentes a versão quântica do interferômetro de Mach-Zehnder, desde a construção das teias geradoras, passando pela construção dos conjuntos coerentes e correspondente transição dos estados associada.

\footnotetext{
${ }^{1}$ rafaelbba@ucpel.tche.br

2reiser@ucpel.tche.br

${ }^{3}$ rocha@ucpel.tche.br
} 
Os conceitos básicos de espaços coerentes são descritos na Seção 2. A visão geral do modelo qGM, incluindo a construção dos estados e processos é apresentada na Seção 3. O interferômetro de Mach-Zehnder é estudado na Seção 4. e, posteriormente, apresenta-se a interpretação no modelo de circuitos (Seção 4.1.) e no modelo qGM (Seção 4.2.). As conclusão resumem os resultados alcançados.

\section{Espaços Coerentes ou Domínios de Girard}

Uma teia $\mathbf{W}=\left(W, \approx_{W}\right)$ é um par consistindo de um conjunto $W$ com uma relação reflexiva e simétrica $\approx_{W}$. Todo subconjunto da teia com pares de elementos consistentes pela relação de coerência $\approx_{w}$ é um subconjunto coerente. A coleção de subconjuntos coerentes da teia $\mathbf{W}$, ordenada pela relação de inclusão define um espaço coerente $\mathbb{W} \equiv(\operatorname{Coh}(\mathbf{W}), \subseteq)$. Na teoria dos espaços coerentes, uma função estável é uma função contínua no sentido de Scott[15], que satisfaz às propriedades de estabilidade, garantindo a existência da menor aproximação finita no conjunto imagem $[17,1]$. Um elemento finito (compacto) de $\mathbb{W}$ é um elemento $a \in \mathbb{W}$ tal que, para qualquer subconjunto dirigido $\mathcal{X} \in \mathbb{W}$, se $a \subseteq \bigcup \mathcal{X}$ então existe $x \in \mathcal{X}$ tal que $a \subseteq x$. $\mathbb{W}_{\text {fin }}$ indica o conjunto dos subconjuntos coerentes finitos de $\mathbb{W}$.

Seja o grafo não dirigido $\mathbf{A} \multimap \mathbf{B}=\left(A \times B, \approx_{-}\right)$, onde a relação de coerência é dada pela expressão:

$$
(\alpha, \beta) \approx_{\multimap}\left(\alpha^{\prime}, \beta^{\prime}\right) \leftrightarrow\left\{\begin{array}{l}
\alpha \approx_{\mathbf{A}} \alpha^{\prime} \rightarrow \beta \approx_{\mathbf{B}} \beta^{\prime} \text { e } \\
\left(\neg\left(\beta \approx_{\mathbf{B}} \beta^{\prime}\right) \vee\left(\beta=\beta^{\prime}\right)\right) \rightarrow\left(\neg\left(\alpha \approx_{\mathbf{A}} \alpha^{\prime}\right) \vee\left(\alpha=\alpha^{\prime}\right)\right) .
\end{array}\right.
$$

O domínio $\mathbb{A} \multimap \mathbb{B} \equiv(\operatorname{Coh}(\mathbf{A} \multimap \mathbf{B}), \subseteq)$ dos traços lineares de função de $\mathbb{A}$ para $\mathbb{B}$ é definido pela coleção de conjuntos coerentes em $\mathbf{A} \multimap \mathbf{B}$, ordenada pela relação de inclusão.

A soma direta entre $\mathbb{A}$ e $\mathbb{B}$ define o espaço coerente $\mathbb{A} \amalg \mathbb{B}=(\operatorname{Coh}(\mathbf{A} \coprod \mathbf{B})$, $\subseteq)$, onde tem-se que a teia $\mathbf{A} \amalg \mathbf{B}=\left(A \cup \dot{\cup} B, \approx_{\amalg}\right)$ é dada pela união disjunta dos conjuntos $A$ e $B$ e pela relação de coerência:

$$
(0, \alpha) \approx_{\amalg}\left(0, \alpha^{\prime}\right) \Leftrightarrow \alpha \approx_{A} \alpha^{\prime} \quad \text { e } \quad(1, \beta) \approx_{\amalg}\left(1, \beta^{\prime}\right) \Leftrightarrow \beta \approx_{B} \beta^{\prime} .
$$

$\mathrm{O}$ produto direto entre $\mathbb{A} \mathrm{e} \mathbb{B}$, indicado pela expressão $\mathbb{A} \prod \mathbb{B}=\left(\operatorname{Coh}\left(\mathbf{A} \prod \mathbf{B}\right)\right.$, $\subseteq)$, é gerado pelo grafo $\mathbf{A} \prod \mathbf{B}=\left(A \cup \dot{B} B, \approx_{\Pi}\right)$ e pela coerência:

$(0, \alpha) \approx_{\Pi}\left(0, \alpha^{\prime}\right) \Leftrightarrow \alpha \approx_{A} \alpha^{\prime}, \quad(1, \beta) \approx_{\Pi}\left(1, \beta^{\prime}\right) \Leftrightarrow \beta \approx_{B} \beta^{\prime} \quad$ e $\quad(0, \alpha) \approx_{\Pi}(1, \beta)$.

\section{Modelo qGM}

Primeiro, as noções de valores de memória e posição de memória são considerados, introduzindo os principais conceitos do domínio de estados do modelo qGM. 


\subsection{Posições e Valores de Memória}

Seja a sequência de subconjuntos que correspondem à expansão diádica das bases canônicas para sistemas quânticos de dimensões finitas:

$$
\begin{aligned}
& Q=\{0 ., 1 .\}, \quad Q^{2}=\{0.0,0.1,1.0,1.1\}, \ldots \\
& Q^{n}=\left\{0.0,0.1, \ldots, 0.1^{n-1}, 1.0,1.1, \ldots, 1.1^{n-1}\right\} .
\end{aligned}
$$

Na Eq. 3.1, o conjunto $Q^{n}$ representa a expansão diádica da base canônica para sistemas quânticos de dimensão finita ( $n$ qubits ou $2^{n}$ bits).

Considera-se a ordem (transfinita) do tipo $2 \omega$, introduzida em [16], cuja notação para a sequência ordenada de seus elementos é dada por: $0,1,2, \ldots, \omega, \omega+1, \omega+$ $2, \ldots 2 \omega$.

Na expansão diádica dos ordinais transfinitos $2 \omega$, indicada por $Q^{w}$, verificam-se as inclusões $Q \subseteq Q^{2} \subseteq \ldots \subseteq Q^{n} \subseteq Q^{n+1} \subseteq \ldots \subseteq Q^{w}$. O conjunto $Q^{\omega}$ caracteriza as posições (endereços) da memória quântica, global e possivelmente infinita, associada ao modelo qGM. Fixada um alfabeto binário $(\{0,1\},\{+,-\},\{\uparrow, \downarrow\})$ para representação dos vetores (infinitos) do espaço de Hilbert, cada elemento em $Q^{\omega}$ identifica a posição de um vetor componente da base computacional.

Seja $\mathcal{C}$ o conjunto de valores de memória (subconjunto dos números complexos normalizados). Cada valor de memória $\alpha=\rho e^{i \theta} \in \mathcal{C}$, representado pelo par de números reais $(\rho, \theta)$, com módulo $\rho \in U=[0,1]$ e arco $\theta, 0 \leq \theta \leq 2 \pi$, satisfaz a condição de normalização:

$$
\alpha=\rho e^{i \theta} \in \mathcal{C}, \alpha \equiv(\rho, \theta) \quad \rightarrow \quad|\alpha|^{2}=\rho^{2} \leq 1 .
$$

\subsection{Domínio de Estados}

Sejam os domínios planos de posições de memória e de valores de memória, indicados pelas expressões $\mathbb{Q}^{\omega} \equiv\left(\operatorname{Coh}\left(Q^{\omega},=_{Q^{\omega}}\right), \subseteq\right)$ e $\mathbb{C} \equiv\left(\operatorname{Coh}\left(\mathcal{C},=_{C}\right), \subseteq\right)$, respectivamente. Considera-se a coleção $S=\operatorname{Coh}\left(\mathbb{Q}^{\omega} \multimap \mathbb{C}\right)$ de subconjuntos coerentes do espaço coerente $\mathbb{Q}^{\omega} \multimap \mathbb{C}$ de traços lineares de funções de $\mathbb{Q}$ para $\mathbb{C}$. O conjunto vazio, "botton", interpreta a função totalmente indefinida; os subconjuntos unitários são objetos parciais e interpretam funções com apenas uma posição definida no conjunto de valores; as funções totalmente definidas são objetos totais em $\mathbb{Q}^{\omega} \multimap \mathbb{C}$.

Definition 3.1 (Função de Normalização). Seja $S=C o h\left(\mathbb{Q}^{\omega} \multimap \mathbb{C}\right)$ a coleção dos traços lineares de funções de $\mathbb{Q}$ para $\mathbb{C}$. A função \|\|$:$ Coh $\left(\mathbb{Q}^{\omega} \multimap \mathbb{C}\right) \rightarrow[0,1]$ é definida pela expressão

$$
\|s\|=\sum_{\left\{\alpha^{n}\right\} \in s}\left|\alpha^{n}\right|^{2} \leq 1,
$$

Para cada traço linear $s,|s|$ retorna uma aproximação para a norma (euclidiana) obtidas dos valores de memória já definidos em cada estado. Estes valores de memória correspondem aos escalares associados à combinação linear dos vetores componentes da base computacional do espaço de Hilbert.

Definition 3.2 (Função de Posição). Seja $S=\operatorname{Coh}\left(\mathbb{Q}^{\omega} \multimap \mathbb{C}\right)$ a coleção dos traços lineares de funções de $\mathbb{Q}$ para $\mathbb{C}$. A função $\Upsilon: \operatorname{Coh}\left(\mathbb{Q}^{\omega} \multimap \mathbb{C}\right) \rightarrow \wp\left(Q^{\omega}\right)$ é definida pela expressão 


$$
\Upsilon(s)=\left\{n \mid\left\{\alpha^{n}\right\} \subseteq s\right\} .
$$

Para cada traço linear $s, \Upsilon(s)$ retorna o conjunto de posições já definidas em cada estado $(s(\alpha) \neq \perp)$, as quais representam os de vetores da base computacional, neste caso com coordenadas já definidas para cada estado.

Ao introduzir as funções de normalização e de posição, Definições 3.1 e 3.2, respectivamente, a ortogonalidade entre vetores da base computacional é garantida pela compatibilidade binária. A condição de normalidade é definida sobre todos os vetores, estendendo esta noção sobre conjuntos coerentes de traços lineares em $S$. Entretanto, preserva-se a coerência entre todos os tokens, obtida por valores associados a posições de memória definidas.

Definition 3.3 (Definição de Estado de Memória). Seja $S=\operatorname{Coh}\left(\mathbb{Q}^{\omega} \multimap \mathbb{C}\right) a$ coleção dos traços lineares de funções de $\mathbb{Q}$ para $\mathbb{C}$. Os elementos $s \in S$ que satisfazem a condição:

$$
|s|=1 \rightarrow \Upsilon(s)=Q^{\omega},
$$

ordenados pela relação de inclusão definem o domínio qualitativo dos estados computacionais, indicado pela expressão $\mathbb{S}=(\operatorname{Coh}(S), \subseteq)$.

Assim, pela Definição 3.3 para um subconjunto coerente $s \in C o h\left(\mathbb{Q}^{\omega} \multimap \mathbb{C}\right)$, tal que $s=\left\{\alpha^{0.0}, \beta^{1.0}\right\}$, tem-se que $\|s\|=|\alpha|^{2}+|\beta|^{2}=1$ provê interpretação para o vetor $(\alpha, 0, \ldots, \beta, 0, \ldots) \in l_{\omega}(\mathcal{H}$, cuja projeção $(\alpha, \beta)$ pertence ao espaço de Hilbert bidimensional $\left(l_{2}(\mathcal{H})\right)$.

\subsection{Processos no Modelo qGM}

No modelo qGM considera-se um processo computacional $\mathbf{p}$ como definido por ações(operações) indexados por pontos de um espaço geométrico obtido a partir dos ordinais transfinitos. A indexação de processos é fundamental para a formalização da base computacional, na qual são definidas as relações que satisfazem as condições de concorrência síncrona (associada ao paralelismo quântico), e de conflito de acesso a memória (o não-determinismo associado a operação de medida). As principais características reescritas logo a seguir, foram obtidas em [11, 12].

A construção do processo computacional $\mathbf{p}$ modelando as portas quânticas unitárias é obtida pela aplicação de construtores (produtos seqüencial e paralelo e somas determinísticas ou não-determinísticas). O modelo caracteriza cada processo computacional p como uma transformação de estados de computação [15], ou seja, a idéia básica é obter a representação dos tipos de dados de entrada e de saída como conjuntos parcialmente ordenados, denominados espaços coerentes, e as computações são representadas através de funções entre esses domínios.

Pela noção de aproximação, definida pela relação de inclusão entre subconjuntos coerentes, tem-se a construção dos objetos (totais) do espaço coerente de processos, gerando informação coerente. Na representação de processos em sistemas de $n$ qubits, considera-se que $2^{n}$ posições da memória possam ser simultaneamente alteradas, modelando o paralelismo quântico. Consideram-se a seguir, apenas as definições relacionadas com os primeiros níveis da estrutura ordenada, visando interpretação do interferômetro de Mach-Zehnder. 


\subsection{Domínio de Processos}

Todo processo quântico é definido como a sincronização de processos elementares, descrevendo uma transição entre estados quânticos puros (determinísticos), executada em uma unidade de tempo computacional (1utc). Em [12], considera-se um processo elementar como uma função $\mathrm{d}^{(k)}$ satisfazendo a proposição:

Proposition 3.1 (Processos Elementares Clássicos). Seja $\mathbb{A} \equiv\left[\mathbb{Q}^{\omega} \multimap \mathbb{C}_{N}\right] \multimap$ $\mathbb{C}_{N}$ o espaço coerente das ações (operações) computacionais. Se d, $\mathrm{pr}^{(i . k)} \in \mathbb{A}$, com $\operatorname{pr}^{(i . k)}(s)=s(i . k)$, verificam-se as propriedades de continuidade, estabilidade e linearidade para a função $\mathrm{d}^{(i . k)} \in\left[\mathbb{Q}^{\omega} \multimap \mathbb{C}_{N}\right] \rightarrow\left[\mathbb{Q}^{\omega} \multimap \mathbb{C}_{N}\right]$ definida pela expressão

$$
\mathrm{d}^{(i . k)}(s)(j . l)= \begin{cases}\operatorname{pr}^{(j . l)}(s)=s(j . l) & \text { se } l \neq k, \\ \mathrm{~d}(s) & \text { se } l=k .\end{cases}
$$

A coleção de todas as funções lineares satisfazendo a Prop. 3.1, define o conjunto de processos elementares, permitindo o conflito no acesso (leitura) aos dados. Entretanto, processos elementares só podem escrever em uma posição na memória global. Isto significa que computações síncronas são definidas pelo produto paralelo de processos elementares que ocorrem simultaneamente. Neste caso, cada posição de memória é transformada por exatamente um processo elementar no modelo qGM, em 1utc.

Definition 3.4 (Domínio de Processos Quânticos Elementares). Seja $\mathbf{D}_{0} \equiv\left(\mathcal{D}_{0},=\right.$ ) a teia discreta formada por processos elementares, de acordo com Def 3.1. Neste caso, $\mathbb{D}_{0} \equiv\left(\operatorname{Coh}\left(\mathbf{D}_{0}\right), \subseteq\right)$ indica o espaço coerente plano dos processos elementares. Tem-se que Coh $\left(\mathbf{D}_{0}\right)=\{\emptyset\} \cup\left\{\left\{u^{(k)}\right\} \mid u^{(k)} \in \mathcal{D}_{0}\right\}$.

Definition 3.5 (Funções Posição-processo e Magnitude-processo). Considere o dominio $\mathbb{D}_{0}$ dos processos elementares e as funções posição-processo e magnitudeprocesso, respectivamente definidas pelas expressões:

- $\Upsilon_{0}: \mathbf{D}_{0} \rightarrow \wp(Q), \Upsilon_{0}(x)=\alpha \mid \alpha^{i} \in x$;

- ||$_{0}: \mathbf{D}_{0} \rightarrow U,|x|_{0}=\sum_{\alpha^{i} \in x}|\alpha|$

Definition 3.6 (Domínio de Processos Quânticos Elementares). Seja $\mathbb{D}_{0}=\left(\operatorname{Coh}\left(\mathbb{D}_{0}\right)\right.$, $\subseteq)$ o espaço coerente dos processos clássicos elementares. Sejam $x, y \in \operatorname{Coh}\left(\mathbb{D}_{0}\right)$ subconjuntos coerentes satisfazendo a relação de coerência

$$
x \approx y \leftrightarrow \Upsilon_{0}(x) \cap \Upsilon_{0}(y)=\emptyset \quad \text { e } \quad|x|,|y| \leq 1 .
$$

A coleção de subconjuntos coerentes $\left(\left(\operatorname{Coh}\left(\mathbb{D}_{0}\right), \approx\right)\right)$, ordenada pela inclusão, define o domínio $\overline{\mathbb{D}}_{0}=\left(\left(\operatorname{Coh}\left(\mathbb{D}_{0}\right), \approx\right), \subseteq\right)$ dos processos quânticos elementares.

Pela Def. 3.6, o domínio $\overline{\mathbb{D}}_{0}$ interpreta as transformações unitárias sobre sistemas quânticos de 1 qubit, garantindo (pela Eq.(3.2)) a sincronização de ações ortogonais, inversíveis e que também preservam a condição de normalidade. Na construção dual, tem-se a interpretação das operações de medidas.

O operador produto direto [1], aplicado sobre o espaço coerentes de processos quânticos $\overline{\mathbb{D}_{0}}$, indicado pela expressão $\overline{\mathbb{D}_{0}} \prod \overline{\mathbb{D}_{0}}$, prove interpretação para o produto 
seqüencial de processos quânticos. A composição de processos seqüenciais é modelada pela iteração do operador produto direto. Assim, novos níveis da estrutura ordenada são definidos, como a soma direta $\mathbb{D}_{1}=\overline{\mathbb{D}_{0}} \amalg\left(\overline{\mathbb{D}_{0}} \prod \overline{\mathbb{D}_{0}}\right)$. O domínio $\mathbb{D}_{\infty}$ é obtido pelas sucessivas aplicações dos functores (produto direto e soma direta) sobre primeiro nível $\mathbb{D}_{0}-\mathbb{D}_{1}$.

As demais etapas da construção indutiva do espaço coerente dos processos $\mathbb{D}_{\infty}$ está descrita em [11] [12], incluindo o processo de completação.

\section{Fenômeno da Interferência}

Este trabalho considera o Interferômetro de Mach-Zehnder (IMZ), por tratar-se de instrumento análogo ao experimento de dupla fenda [8, 14], que apresenta uma forma simplificada e atual do fenômeno de interferência, incluindo uma simulação virtual apresentada em [13, 9] e denominada Interferômetro Virtual de Mach-Zehnder. No IMZ, os principais conceitos envolvidos são a introdução de uma mudança de fase entre diferentes caminhos ópticos e a superposição das ondas defasadas. A versão quântica da porta óptica IMZ está baseada na diminuição da intensidade do feixe, induzindo a emissão intermitente de fótons $[2,14]$. Assim, tornam-se necessários detectores suficientemente sensíveis, localizados ao final do aparato, capazes de detectar a presença de fótons únicos (feixes monofotônicos) [10].

$\mathrm{Na}$ estrutura e no funcionamento da porta óptica, graficamente representado como na Figura 1A, tem-se uma entrada para os possíveis caminhos, os quais estão representados por $|0\rangle$ e $|1\rangle$ na notação de Dirac. Verifica-se que o aparato possui dois espelhos semi-refletores (ES1, ES2) e dois refletores (E1,E2). Um espelho semirefletor é um dispositivo que reflete metade da onda de luz incidente, transmitindo a outra metade sem ser afetada, introduzindo diferentes atrasos na propagação ao longo dos caminhos. Considera-se que não ocorre perda quando da ocorrência de reflexão da luz [3].

A presença de defasadores (ou moduladores de fase) $\theta_{0}$ e $\theta_{1}$ pode representar, por exemplo, uma alteração de percurso nos caminhos ópticos do interferômetro, caracterizando uma mudança de fase dos feixes de onda. Na sequência, os feixes de ondas passam novamente por um espelho semi-refletor, para finalmente alcançarem os dispositivos $\mathcal{D}_{0}$ e $\mathcal{D}_{1}$, denotando os detectores da intensidade da onda nas respectivas saídas do interferômetro. Como cada componente da onda incidente se desloca por um caminho distinto, poderíamos esperar que cada detector $\mathcal{D}_{0}$ e $\mathcal{D}_{1}$ medisse $50 \%$ do feixe monofotônico (fóton). Mas o experimento mostra que $100 \%$ do feixe original incide no detector $\mathcal{D}_{1}$ (superposição construtiva de onda em $\mathcal{D}_{1}$ ) e, portanto, em $\mathcal{D}_{0}$ não ocorre registro (resultado gerado pela superposição destrutiva de onda em $\mathcal{D}_{0}$ ).

O interferômetro viabiliza a verificação do comportamento ondulatório associado a uma partícula atômica (fóton) [3], mostrando que esse comportamento ondulatório é mantido, mesmo quando o feixe de entrada é constituído por uma única partícula atômica (fóton) incidente em cada instante. Esta constatação marca o "estranhamento" típico dos processos quânticos: a interferência só seria possível, nesse caso, se o fóton pudesse percorrer os dois caminhos ao mesmo tempo para, no 
final, interferir consigo mesmo.

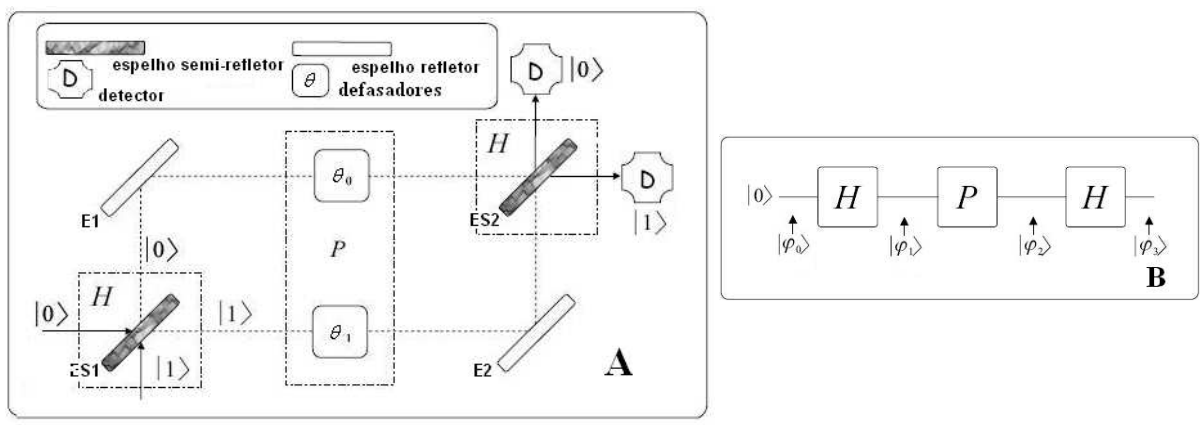

Figura 1: Porta Óptica e Circuito Quântico do Interferômetro de Mach-Zehnder.

\subsection{Interferômetro no Modelo de Circuitos}

Restringe-se o estudo ao espaço de Hilbert bi-dimensional $l_{2}(\mathcal{H} 2)$ e considera-se na base canônica o vetor $\varphi=(\alpha, \beta)^{t} \in \mathcal{H} 2$, com $\alpha, \beta$ números complexos satisfazendo a condição de normalidade $\alpha^{2}+\beta^{2}=1$. As portas quânticas básicas são transformações lineares $U$ (no caso $H$ e $P$ ) definidas por matrizes unitárias de ordem 2, tais que $U^{\dagger} U=U U^{\dagger}=I$, onde $U^{\dagger}$ indica a transposta conjugada da matriz $U$.

Aplica-se a linguagem de circuitos para descrever o processo de interferência sendo a base canônica indicada por $\{|0\rangle,|1\rangle\}$ e um qubit $|\varphi\rangle=\alpha|0\rangle+\beta|1\rangle$, sempre que $\alpha$ e $\beta$ satisfaçam a condição de normalidade. Na evolução temporal, associado à Figura 1B introduzida em [5, 2], faz-se uso de portas quânticas sobre estados de 1 qubit, sendo o estado inicial indicado por $\left|\varphi_{0}\right\rangle$. Cada espelho semi-refletor está representado por uma porta Hadamard $(H)$, e os defasadores $\theta_{0}$ e $\theta_{1}$ são representados pela porta unitária Phase $(P)$. A evolução do sistema pode também ser observada pela transformação dos estados, obtida em etapas a partir do estado inicial $\left|\varphi_{0}\right\rangle$, resultando em três novos estados indicados por $\left|\varphi_{1}\right\rangle,\left|\varphi_{2}\right\rangle$ e $\left|\varphi_{3}\right\rangle$.

Usa-se a notação de Dirac e a interpretação matricial para descrever as etapas de evolução:

1. $\left|\varphi_{1}\right\rangle=H\left|\varphi_{0}\right\rangle$, indicando a aplicação da porta $\operatorname{Hadamard}(\mathrm{H})$ ao estado inicial $\left|\varphi_{0}\right\rangle$, ou na notação matricial, conforme mostra a Equação 4.3:

$$
\frac{1}{\sqrt{2}}\left(\begin{array}{l}
1 \\
1
\end{array}\right)=\frac{1}{\sqrt{2}}\left(\begin{array}{cc}
1 & 1 \\
1 & -1
\end{array}\right)\left(\begin{array}{l}
1 \\
0
\end{array}\right) \text {. }
$$

2. $\left|\varphi_{2}\right\rangle=P\left|\varphi_{1}\right\rangle$, indicando a determinação do estado $\left|\varphi_{2}\right\rangle$ pela ação da porta Phase $(\mathrm{P})$ sobre o estado $\left|\varphi_{1}\right\rangle$, com descrição análoga na expressão matricial da Equação 4.4:

$$
\frac{1}{\sqrt{2}}\left(\begin{array}{c}
e^{i \theta_{0}} \\
e^{i \theta_{1}}
\end{array}\right)=\left(\begin{array}{cc}
e^{i \theta_{0}} & 0 \\
0 & e^{i \theta_{1}}
\end{array}\right) \frac{1}{\sqrt{2}}\left(\begin{array}{l}
1 \\
1
\end{array}\right)
$$




\begin{tabular}{|c|c|c|c|c|c|c|c|c|}
\hline$\left|\varphi_{0}\right\rangle$ & 0 & \multicolumn{2}{|l|}{1} & & $\left|\varphi_{1}\right\rangle$ & 0 & 1 & $\ldots$ \\
\hline 0. & $(1,0)$ & \multicolumn{2}{|l|}{$(0,0)$} & & 0 . & $\left(\frac{1}{\sqrt{2}}, 0\right)$ & $(0,0)$ & $\ldots$ \\
\hline \multirow[t]{4}{*}{1.} & $(0,0)$ & \multicolumn{2}{|l|}{$(0,0)$} & & 1. & $\left(\frac{1}{\sqrt{2}}, 0\right)$ & $(0,0)$ & $\ldots$ \\
\hline & & $\left|\varphi_{2}\right\rangle$ & & 1 & $\ldots$ & & & \\
\hline & & 0 . & $\left(\frac{1}{\sqrt{2}} e\right.$ & $(0,0)$ & . & & & \\
\hline & & 1. & $\left(\frac{1}{\sqrt{2}} e\right.$ & $(0,0)$ & $\ldots$ & & & \\
\hline
\end{tabular}

Figura 2: Representação de $\left|\varphi_{0}\right\rangle,\left|\varphi_{1}\right\rangle,\left|\varphi_{2}\right\rangle$ no modelo qGM.

3. $\left|\varphi_{3}\right\rangle=H\left|\varphi_{2}\right\rangle$, ou ainda, tem-se a determinação do estado $\left|\varphi_{3}\right\rangle$, interpretando a recombinação dos percursos da Fig.1, na Equação 4.5:

$$
\frac{1}{2}\left(\begin{array}{c}
e^{i \theta_{0}}+e^{i \theta_{1}} \\
e^{i \theta_{0}}-e^{i \theta_{1}}
\end{array}\right)=\frac{1}{\sqrt{2}}\left(\begin{array}{cc}
1 & 1 \\
\frac{1}{\sqrt{2}} & -1
\end{array}\right) \frac{1}{\sqrt{2}}\left(\begin{array}{c}
e^{i \theta_{0}} \\
e^{i \theta_{1}}
\end{array}\right) .
$$

Por simplificação, considera-se a fatoração da Eq. (4.5), resultando em:

$$
\left|\varphi_{3}\right\rangle=\frac{1}{2} e^{\frac{i}{2}\left(\theta_{0}+\theta_{1}\right)}\left(\begin{array}{c}
e^{\frac{i}{2}\left(\theta_{0}-\theta_{1}\right)}-e^{\frac{-i}{2}\left(\theta_{0}-\theta_{1}\right)} \\
e^{\frac{i}{2}\left(\theta_{0}-\theta_{1}\right)}+e^{\frac{-i}{2}\left(\theta_{0}-\theta_{1}\right)}
\end{array}\right) .
$$

Substituindo-se as exponenciais pelas funções trigonométricas, reduz-se a Eq. (4.6) à forma

$$
\left|\varphi_{3}\right\rangle=e^{\frac{i}{2}\left(\theta_{0}+\theta_{1}\right)}\left(i \sin \left(\frac{\theta_{0}-\theta_{1}}{2}\right)|0\rangle+\cos \left(\frac{\theta_{0}-\theta_{1}}{2}\right)|1\rangle\right) .
$$

Omitindo-se a expressão $e^{\frac{i}{2}\left(\theta_{0}+\theta_{1}\right)}$ e considerando $\frac{\Delta \theta}{2}=\frac{\theta_{0}-\theta_{1}}{2}$, a Eq. (4.7) reduz-se à expressão:

$$
\left|\varphi_{3}\right\rangle=i \sin \left(\frac{\triangle \theta}{2}\right)|0\rangle+\cos \left(\frac{\triangle \theta}{2}\right)|1\rangle .
$$

4. Pela observação da Equação (4.8), verificam-se as seguintes probabilidades:

- se $P_{0}=\sin ^{2}\left(\frac{\triangle \theta}{2}\right)$, e $\triangle \theta=0$ então $P_{0}=0$,

- se $P_{1}=\cos ^{2}\left(\frac{\Delta \theta}{2}\right)$, e $\triangle \theta=0$ então $P_{1}=1$.

A aplicação de um feixe monofotônico no IMZ mostra que este irá interagir consigo mesmo, produzindo interferência construtiva num dos detectores, e interferência destrutiva no outro detector.

\subsection{Interferômetro no Modelo qGM}

Consideram-se os resultados da Seção 3. para obter uma interpretação para o IMZ introduzido na Seção 4., segundo as abstrações do modelo qGM. 


\subsubsection{Interpretação de Estados}

Cada estado $\left|\varphi_{i}\right\rangle_{0 \leq i \leq 3}$ está associado, no modelo qGM, ao traço linear de uma função $\mathbf{s}_{i 0 \leq i \leq 3}$, em $\mathbb{S}_{I}$, o subespaço coerente de estados quânticos associado ao IMZ. Tem-se que $\mathbb{S}_{I} \subseteq \mathbb{Q}^{\omega} \multimap \mathbb{C}$ e, cada conjunto coerente $s \in \mathbb{S}_{I}$ corresponde ao traço linear de $\mathbf{s}: Q^{\omega} \rightarrow \mathbb{C}$, ou seja, $s=\operatorname{ltr}(\mathbf{s}) \in \mathbb{Q}^{\omega} \multimap \mathbb{C}$.

Assim, um conjunto coerente $s \in \mathbb{S}_{I}$ é um conjunto de pares ordenados $\alpha^{n} \equiv$ $(\rho, \theta)^{n} \in s$, sempre que $\mathbf{s}(n)=\alpha \in \mathbb{C}$, o qual possui no máximo duas posições $(0.0$ e 1.0) associadas a valores de memória não-nulos ${ }^{4}$, ou ainda, $\operatorname{ltr}(\mathbf{s}) \equiv\left\{\alpha^{0.0}, \beta^{0.1}\right\}$ sempre que $\left\{|\alpha|^{2}+|\beta|^{2} \leq 1\right\}$.

Nesta interpretação, considere os subconjuntos coerentes que são maximais para a função de normalização e para a função de posição (objetos totais), $\operatorname{ltr}\left(\varphi_{i}\right)_{0 \leq i \leq 3}=$ $\left\{\alpha_{i}^{0.0}, \beta_{i}^{0.1}\right\}_{\left\{\left|\alpha_{i}\right|^{2}+\left|\beta_{i}\right|^{2}=1\right\}}$ :

$$
\begin{aligned}
& \text { - } l \operatorname{tr}\left(\varphi_{0}\right)=\left\{(1,0)^{0.0},(0,0)^{1.0}\right\} \\
& \text { - } \operatorname{tr}\left(\varphi_{1}\right)=\left\{\left(\frac{1}{\sqrt{2}}, 0\right)^{0.0},\left(\frac{1}{\sqrt{2}}, 0\right)^{1.0}\right\} \\
& \text { - } l \operatorname{tr}\left(\varphi_{2}\right)=\left\{\left(\frac{1}{\sqrt{2}} e^{i \theta_{0}}, 0\right)^{0.0},\left(\frac{1}{\sqrt{2}} e^{i \theta_{1}}, 0\right)^{1.0}\right\} \\
& \text { - } l \operatorname{tr}\left(\varphi_{3}\right)=\left\{\left(\sin \left(\frac{\triangle \theta}{2}\right), \frac{\pi}{2}\right)^{0.0},\left(\cos \left(\frac{\triangle \theta}{2}\right), 0\right)^{1.0}\right\} .
\end{aligned}
$$

Em particular, na Figura 2, representa-se a função $\varphi_{1}: \mathbb{Q}^{\omega} \rightarrow \mathbb{C}$ cujo traço linear interpreta a superposição $\left|\varphi_{1}\right\rangle$. Salientam-se os subconjuntos coerentes nãovazios $\left\{\left(\frac{1}{\sqrt{2}}, 0\right)^{0.0}\right\}$ e $\left\{\left(\frac{1}{\sqrt{2}}, 0\right)^{1.0}\right\}$, os quais interpretam estados parciais do estado $\left\{\left(\frac{1}{\sqrt{2}}, 0\right)^{0.0},\left(\frac{1}{\sqrt{2}}, 0\right)^{1.0}\right\}$, os quais são aproximações não maximais para a condição de normalização. Tem-se que:

- $\left\{\left(\frac{1}{\sqrt{2}}, 0\right)^{0.0}\right\}$ corresponde ao traço linear da função que ainda está indefinida na posição 1.0, ou seja, tem-se que a primeira componente da superposição $\left|\varphi_{1}\right\rangle$ recebe o valor $\left(\frac{1}{\sqrt{2}}, 0\right)$ e todas as demais posições tem valor $(0,0)$, exceto a posição indefinida. Logo o conjunto coerente $\left\{\left(\frac{1}{\sqrt{2}}, 0\right)^{0.0}\right\}$ interpreta o estado parcial $\frac{1}{\sqrt{2}}|0\rangle+\perp|1\rangle$ (veja representação na Figura 4.)

- $\left\{\left(\frac{1}{\sqrt{2}}, 0\right)^{1.0}\right\} \subseteq\left\{\left(\frac{1}{\sqrt{2}}, 0\right)^{0.0},\left(\frac{1}{\sqrt{2}}, 0\right)^{1.0}\right\}$ corresponde ao traço linear da função que ainda está indefinida na posição 0.0. Neste caso, o conjunto coerente $\left\{\left(\frac{1}{\sqrt{2}}, 0\right)^{1.0}\right\}$ interpreta o estado parcial $\perp|0\rangle+\frac{1}{\sqrt{2}}|1\rangle$.

De forma análoga, obtém-se os estados parciais de $\left|\varphi_{2}\right\rangle$, apresentados nos distintos caminhos do interferômetro da Figura 4 com as seguintes interpretações:

- $\left\{\left(\frac{1}{\sqrt{2}} e^{i \theta_{0}}, 0\right)^{0.0}\right\}$ interpreta $\left(\frac{1}{\sqrt{2}} e^{i \theta_{0}}, 0\right)|0\rangle+\perp|1\rangle$;

- $\left\{\left(\frac{1}{\sqrt{2}} e^{i \theta_{1}}, 0\right)^{1.0}\right\}$ interpreta $\perp|0\rangle+\left(\frac{1}{\sqrt{2}} e^{i \theta_{1}}, 0\right)|1\rangle$.

Por fim, esta análise estende-se ao $\operatorname{ltr}\left(s_{3}\right)$ e seus conjuntos coerentes que interpretam as aproximações para o estado $\left|\varphi_{3}\right\rangle$, de tal forma a completar a interpretação para os distintos caminhos do IMZ segundo as abstrações do modelo qGM. A função $s_{3}$, e sua correspondente representação como estado de memória no modelo qGM, estão na Figura 3.

\footnotetext{
${ }^{4}$ Pares com valores de memória nulos serão omitidos na representação, quando não relevantes no contexto em estudo
} 
- $\left\{\left(\sin \left(\frac{\triangle \theta}{2}\right), \frac{\pi}{2}\right)^{0.0}\right\}$ interpreta $i\left(\sin \left(\frac{\triangle \theta}{2}\right)\right)|0\rangle+\perp|1\rangle$;

- $\left\{\left(\cos \left(\frac{\triangle \theta}{2}\right), 0\right)^{1.0}\right\}$ interpreta $\perp|0\rangle+\left(\cos \left(\frac{\triangle \theta}{2}\right)\right)|1\rangle$.

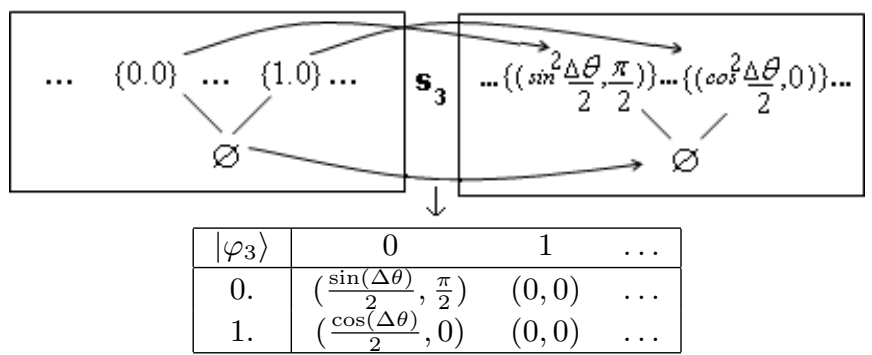

Figura 3: Interpretação do estado $\varphi_{3}$ no modelo qGM.

\subsection{2. $\quad$ Interpretação de Processos}

Define-se, nesta seção o procedimento de construção dos espaços coerentes relacionados com o interferômetro, bem como sua correspondência com o modelo de circuitos $[6,14,2,10]$. O conceito mais fundamental nesta seção é o de processo elementar, o qual pode ser descrito como uma transição entre estados clássicos, executada em uma unidade de tempo computacional (1utc).

Seja $\operatorname{pr}^{i . j}$ a notação para função projeção na posição de memória $i . j \in Q^{\omega}$. Para interpretação das portas $H$ e $P$ no interferômetro, consideram-se as seguintes operações clássicas:

$$
\begin{gathered}
\bullet \mathrm{h}^{(0.0)}(s)(k)=\left\{\begin{array}{l}
s(1.0):=\operatorname{pr}^{(1.0)}(s)=s(1.0), \\
s(0.0):=\left\{\frac{1}{\sqrt{2}} s(0.0)+\frac{1}{\sqrt{2}} s(1.0)\right\},
\end{array}\right. \\
\bullet \mathrm{h}^{(1.0)}(s)(k)=\left\{\begin{array}{l}
s(0.0):=\operatorname{pr}^{(0.0)}(s)=s(0.0), \\
s(1.0):=\left\{\frac{1}{\sqrt{2}} s(0.0)-\frac{1}{\sqrt{2}} s(1.0)\right\}
\end{array}\right. \\
\bullet \mathrm{p}^{(0.0)}(s)(k)=\left\{\begin{array}{l}
s(1.0):=\operatorname{pr}^{(1.0)}(s)=s(1.0), \\
s(0.0):=e^{i \theta_{0}} s(0.0), \\
s(0.0):=\operatorname{pr}^{(0.0)}(s)=s(0.0), \\
s(1.0):=e^{i \theta_{1}} s(0.0)
\end{array}\right.
\end{gathered}
$$

Aplicando-se a Def. 3.6, tem-se que $H^{0}=\left\{\mathrm{h}^{(0.0)}, \mathrm{h}^{(1.0)}\right\} \in \mathbb{D}_{1}$ é o subconjunto coerente interpretando a porta Hadamard, quando aplicada na posição de memória quântica 0 (primeiro qubit) no modelo qGM. Os correspondentes objetos parciais são: 


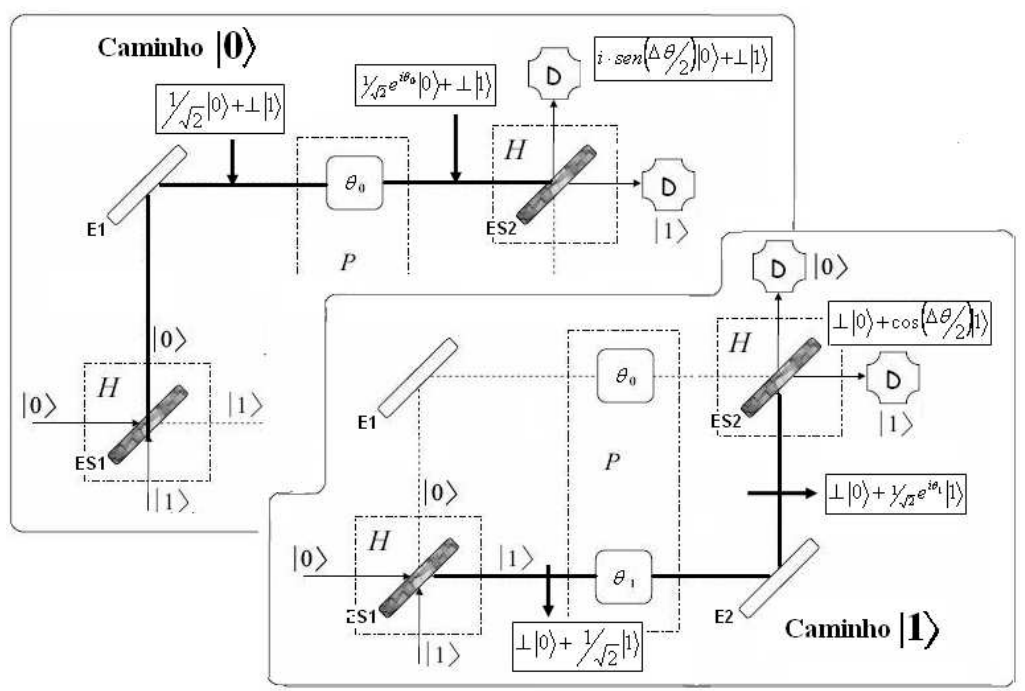

Figura 4: Circuitos ópticos parciais do interferômetro.

- $\left\{\mathrm{h}^{(0.0)}\right\} \in \mathbb{D}_{1}$ interpreta $H_{\perp}=\left(\begin{array}{cc}\frac{1}{\sqrt{2}} & \frac{1}{\sqrt{2}} \\ \perp & \perp\end{array}\right)$

- $\left\{\mathrm{h}^{(1.0)}\right\} \in \mathbb{D}_{1}$ interpreta $H^{\perp}=\left(\begin{array}{cc}\perp & \perp \\ \frac{1}{\sqrt{2}} & \frac{1}{\sqrt{2}}\end{array}\right)$

- $\left\{\mathrm{p}^{(0.0)}\right\} \in \mathbb{D}_{1}$ interpreta $P_{\perp}=\left(\begin{array}{cc}e^{\sqrt{\theta_{0}}} & 0 \\ \perp & \perp\end{array}\right)$

- $\left\{\mathrm{p}^{(1.0)}\right\} \in \mathbb{D}_{1}$ interpreta $P^{\perp}=\left(\begin{array}{cc}\perp & \perp \\ 0 & e^{i \theta_{1}}\end{array}\right)$.

Além disso, tem-se uma interpretação para a evolução dos estados, percorrendo independentemente, cada um dos caminhos. No caso do percurso $|0\rangle$, tem-se:

1. $\left|\varphi_{1}\right\rangle_{\perp}=H_{\perp}\left|\varphi_{0}\right\rangle=\frac{1}{\sqrt{2}}|0\rangle+\perp|1\rangle \Rightarrow\left(\begin{array}{c}\frac{1}{\sqrt{2}} \\ \perp\end{array}\right)=\left(\begin{array}{cc}\frac{1}{\sqrt{2}} & \frac{1}{\sqrt{2}} \\ \perp & \perp\end{array}\right)\left(\begin{array}{l}1 \\ 0\end{array}\right)$.

2. $\left|\varphi_{2}\right\rangle_{\perp}=P_{\perp}\left|\varphi_{1}\right\rangle_{\perp}=\frac{1}{\sqrt{2}} e^{i \theta_{0}}|0\rangle+\perp|1\rangle \Rightarrow\left(\begin{array}{c}\frac{e^{i \theta_{0}}}{\sqrt{2}} \\ \perp\end{array}\right)=\left(\begin{array}{cc}e^{i \theta_{0}} & 0 \\ \perp & \perp\end{array}\right)\left(\begin{array}{c}\frac{1}{\sqrt{2}} \\ \perp\end{array}\right)$.

3. $\left|\varphi_{3}\right\rangle_{\perp}=H_{\perp}\left|\varphi_{2}\right\rangle=i \operatorname{sen} \frac{\Delta \theta}{2}|0\rangle+\perp|1\rangle \Rightarrow\left(\begin{array}{c}\frac{1}{\sqrt{2}} e^{i \theta_{0}} \\ \perp\end{array}\right)=\left(\begin{array}{cc}\frac{1}{\sqrt{2}} & \frac{1}{\sqrt{2}} \\ \perp & \perp\end{array}\right)\left(\begin{array}{c}\frac{1}{\sqrt{2}} e^{i \theta_{0}} \\ \frac{1}{\sqrt{2}} e^{i \theta_{1}}\end{array}\right)$.

Assim, o modelo qGM provê uma representação para as computações parciais, e viabiliza uma interpretação do algoritmo em diferentes contextos. Ou ainda, o caso de estudo baseado no IMZ mostra que os diferentes percursos (rotulados por $|0\rangle$ e $|1\rangle$, indicando as direções no espaço bi-dimensional da base canônica) percorridos por um fóton (qubit), podem ser descritos a partir da interpretação obtida. A seguir, salientam-se as principais observações obtidas: 
- Interpretações sobre o caminho $|0\rangle$.

- A sequência de operações executadas no contexto do percurso $|0\rangle$ é dada pela combinação dos operadores: $H_{\perp} \circ P_{\perp} \circ H_{\perp}$, de acordo com a Figura 5;

- A transformação de estados gerados quando da execução do algoritmo, considerando o contexto do percurso $|0\rangle$, é descrita pela sequência: $|0\rangle$, $\frac{1}{\sqrt{2}}(|0\rangle+\perp|1\rangle), \frac{1}{\sqrt{2}}\left(e^{i \theta_{0}}|0\rangle+\perp|1\rangle\right),\left(i \sin \frac{\delta \theta}{2} e^{i \theta_{0}}\right)(|0\rangle+\perp|1\rangle)$.

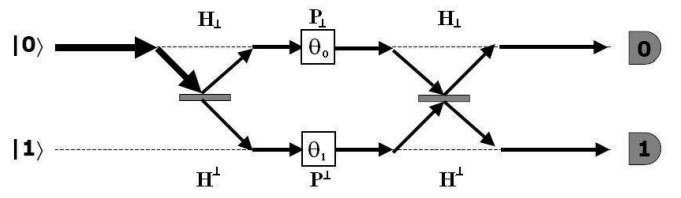

Figura 5: Composição de operadores referentes aos percursos $|0\rangle$ e $|1\rangle$.

- Interpretações sobre o caminho $|1\rangle$.

- A sequência de operações executadas no contexto do percurso $|1\rangle$ é dada pela combinação dos operadores: $H^{\perp} \circ P^{\perp} \circ H^{\perp}$, de acordo com a Figura 6 ;

- A transformação de estados gerados quando da execução do algoritmo, considerando o contexto do percurso $|1\rangle$, esta descrita pelos sequência: $|0\rangle, \frac{1}{\sqrt{2}}(\perp|0\rangle+|1\rangle), \frac{1}{\sqrt{2}}\left(\perp|0\rangle+e^{i \theta_{1}}|1\rangle\right),\left(\cos \frac{\delta \theta}{2} e^{i \theta_{1}}\right)(\perp|0\rangle+|1\rangle)$.

$\left|\varphi_{0}\right\rangle$

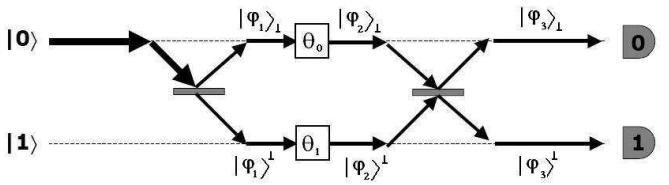

Figura 6: Transformação de estados referentes aos percursos $|0\rangle$ e $|1\rangle$.

\section{Considerações Finais}

O modelo qGM introduz uma interpretação baseada na parcialidade dos objetos que constroem os domínios de estados e de processos para a computação quântica. As abstrações do modelo qGM podem ser aplicadas na interpretação dos algoritmos descritos na linguagem universal de circuitos, com uma adicional abordagem, capaz de mostrar que a evolução de sistemas quânticos, referente às construções síncronas como estados e processos, também pode ser interpretada a partir da análise dos objetos parciais. Esta construção indutiva e uniforme da informação generaliza 
as interpretações já obtidas em sistemas clássicos. A construção dos subconjuntos coerentes que modelam o Interferômetro de Mach-Zehnder pode ser descrita considerando-se diferentes contextos quando da interpretação parcial do estado global, detalhando a composição e construção das portas quânticas como Hadamard e Phase. Também foram consideradas a estrutura ordenada dos conjuntos coerentes sobre os quais se constroem os subespaços coerentes $\mathbb{D}_{0}$ e $\mathbb{D}_{1}$, referentes aos primeiros níveis do domínio $\mathbb{D}_{\infty}$.

Abstract. This work analyzes the interpretations of quantum algorithms in the qGM (Quantum Geometric Machine) model, in order to understand the construction of the information during the process of quantum systems evolution. The ordered structure of the qGM model is able to interpret the construction of the processes and quantum states based on the conception of partial objects, considering the inclusion relationship as the order of information. The work presents a case study, in which the representation of partial objects enables a contextual description for the studied of the Mach-Zehnder Interferometer related to interference phenomena. It shows that such interpretation can not be obtained outside the conceptual outline of the Domain Theory.

\section{Referências}

[1] S. Abramsky, A. Jung, "Domain Theory", Handbook of Logic in Computer Science, Clarendon Press, New York, 1994.

[2] G. Cabral, A. Lima, B. Lula, Interpretando o algoritmo de Deutsch no interferômetro de Mach-Zehnder, Revista Brasileira de Ensino de Física, 26, No. 2 (2004), 97-117.

[3] D. Deutsch, A. Ekert, R. Jozsa, "Concepts of Quantum Computation - The Physics of Quantum Information", Springer-Verlag, 2001.

[4] J. Girard, Between logic and quantic: a tract, in "Linear in Computer Science", Cambridge University Press, 2004.

[5] S. Imre, F. Balazs, "Quantum Computing and Communications", John Wiley \& Sons Inc., 2005.

[6] E. Knill, M.A. Nielsen, "Theory of Quantum Computation", Encyclopaedia of Mathematics, 2002.

[7] A. Lima, B.L. Júnior, "Computação Quântica, Noções Básicas utilizando a Linguagem de Circuitos Quânticos", EDUFCG, Ed. da UFCG, 2007.

[8] M. Nielsen, I. Chuang, "Quantum Computation and Quantum Information", Cambridge University Press, 2000.

[9] F. Ostermann, S. Prado, Interpretações da mecânica quântica em um interferômetro virtual de Mach-Zehnder. Revista Brasileira de Ensino de Física, 27, [S.l.], (2005), 193-203.

[10] O.P. Júnior, "Conceitos de Física Quântica", Editora Livraria da Física, SP, 2003. 
[11] R. Reiser, A. Costa, R. Amaral, Toward Coherence Space-based Models of Quantum Algorithms, in "Proc. of the 2nd Workshop in Nanotecnology and Computation Inspired on Biology", pp. 1-6, PUC-RJ, 2007.

[12] R. Reiser, A. Costa, R. Amaral, Quantum Computing: Computation from Coherence Spaces, in "WEICIQ 2007", Campina Grande: EDUFCG/UFCG, pp.105-114, CG, 2007.

[13] T. Ricci, F. Ostermann, S. Prado, O tratamento clássico do interferômetro de MachZehnder: uma releitura mais moderna do experimento da fenda dupla na introdução da física quântica, Revista Brasileira de Ensino de Física, 29 (2007) 79-88.

[14] E. Rieffel, W. Polak, An introduction to quantum computing for non-physicists, $A C M$ Computing Surveys, 1-45, 1977.

[15] D. Scott, The lattice of flow diagrams, in "Lecture Notes in Mathematics, Symposium on Semantics of Algorithmic Languages", Springer Lecture Note Series, 1971.

[16] R. Stoll, "Set Theory and Logic", Dover Publication Inc., New York, 1961.

[17] A. Troelstra, Lectures on Linear Logic, in "Stanford-CSLI Lecture Notes", 1992. 